\title{
Longitudinal Persistence of Meningeal Enhancement on Postcontrast 7T 3D-FLAIR MRI in Multiple Sclerosis
}

\author{
(D)S.N. Jonas, (DI. Izbudak, (D)A.A. Frazier, and (DD.M. Harrison
}

\begin{abstract}
BACKGROUND AND PURPOSE: Preliminary research has demonstrated that postgadolinium 3D-FLAIR MR imaging at 7T may be a valuable tool for detecting abnormal meningeal enhancement and inflammation in MS; however, researchers have not systematically investigated its longitudinal persistence. We hypothesized that persistence of meningeal enhancement in MS varies on the basis of pattern of enhancement as well as demographic and clinical factors such as treatment status, disease phenotype, and disability score.
\end{abstract}

MATERIALS AND METHODS: Thirty-one subjects with MS were prospectively scanned before and after intravenous contrast administration at 2 time points, approximately 1 year apart. Fifteen subjects in the cohort were scanned at another time approximately 1 year later. Foci of enhancement were categorized into 4 subtypes: subarachnoid spread/fill, subarachnoid nodular, vessel wall, and dural foci. We reviewed follow-up scans to determine whether foci changed between time points and then compared persistence with demographic and clinical variables.

RESULTS: Persistence ranged from $71 \%$ to $100 \%$ at 1 year and $73 \%$ to $100 \%$ at 2 years, depending on the enhancement pattern. Subarachnoid spread/fill and subarachnoid nodular subtypes persisted less often than vessel wall and dural foci. Persistence was not significantly different between those on/off treatment and those with progressive/nonprogressive disease phenotypes. The number of persisting foci was significantly different in subjects with/without increasing Expanded Disability Status Scale scores (median, 12 versus 7.5, $P=.04$ ).

CONCLUSIONS: Longitudinal persistence of meningeal enhancement on 3D-FLAIR at 7T in MS varies by pattern of enhancement and correlates with worsening disability; however, it is not significantly different in those on/off treatment or in those with progressive/ nonprogressive disease phenotypes.

ABBREVIATIONS: EDSS = Expanded Disability Status Scale; MP2RAGE = magnetization-prepared rapid acquistion of 2 gradient echoes

$\mathbf{M}^{\mathrm{s}}$ $\mathrm{S}$ is a chronic inflammatory demyelinating disorder classically affecting white matter within the brain and spinal cord. In the past few decades, an additional pathophysiologic mechanism-meningeal inflammation-has been elucidated in MS, which is now believed to directly contribute to cortical demyeli-

Received March 20, 2018; accepted after revision July 20

From the Department of Radiology (S.N.J., A.A.F.), University of Maryland Medical Center, Baltimore, Maryland; Department of Radiology/Neuroradiology (I.I), Johns Hopkins University, Baltimore, Maryland; and Department of Neurology, University of Maryland School of Medicine (D.M.H.), Baltimore, Maryland.

This study was funded, in part, by grants from EMD Serono and the National Institutes of Health (National Institute of Neurological Disorders and Stroke 1K23NS072366-01A1)

Please address correspondence to Samuel N. Jonas, MD, Department of Radiology, University of Maryland Medical Center, 22 South Greene St, Baltimore, MD 21201; e-mail: samueljonas@umm.edu

-- Indicates open access to non-subscribers at www.ajnr.org

三 Indicates article with supplemental on-line tables.

http://dx.doi.org/10.3174/ajnr.A5796 nation, cortical neuroaxonal loss, microglial activation, and oligodendrocyte dysfunction. ${ }^{1-6}$ Visualization of meningeal inflammation on MR imaging has become an active, and somewhat controversial, area of recent investigation. ${ }^{7}$ Landmark studies have demonstrated that gadolinium-enhanced 3D-FLAIR sequences, which have long been useful for identifying meningeal infection and carcinomatosis, can also be used to image meningeal disease in the MS population. At a magnetic field strength of 3T, Absinta et $\mathrm{al}^{7}$ found that approximately $25 \%$ of patients with MS demonstrated leptomeningeal enhancement on gadolinium-enhanced FLAIR.

Protocols for imaging meningeal enhancement were improved by Zivadinov et al, ${ }^{8}$ who showed the benefit of both preand postcontrast acquisitions and generating subtraction images when assessing meningeal enhancement, because these techniques decrease false-positives and reduce interpretation time. Recent preliminary research has also suggested that 7T MR imaging may be more sensitive than $3 \mathrm{~T}$ for detecting meningeal enhancement. Although no direct 3T-versus-7T comparisons have 
Table 1: MRI sequence parameters

\begin{tabular}{lllllccc}
\hline Sequence & Resolution $(\mathrm{mm})$ & \multicolumn{1}{c}{ TR } & \multicolumn{1}{c}{ TI } & TE & Parallel Imaging & Flip Angle & Time (min:sec) \\
\hline MP2RAGE & $0.7 \times 0.7 \times 0.7$ & $\mathrm{TR}_{\text {volume }}=8.25 \mathrm{~s}$ & $\mathrm{TI}_{1}=1 \mathrm{~s}$ & $1.97 \mathrm{~ms}$ & $\mathrm{SENSE}=2 \times 2$ & $\mathrm{FA}_{1}=7^{\circ}$ & $9: 46$ \\
& & $\mathrm{TR}_{\mathrm{TFE}}=6.9 \mathrm{~ms}$ & $\mathrm{TI}_{2}=3.3 \mathrm{~s}$ & & & $\mathrm{FA}_{2}=5^{\circ}$ & $90^{\circ}$ \\
MPFLAIR & $0.7 \times 0.7 \times 0.7$ & $8000 \mathrm{~ms}$ & $2077 \mathrm{~ms}$ & $400 \mathrm{~ms}$ & SENSE $=2 \times 3$ & $10: 48$ \\
\hline
\end{tabular}

Note:-SENSE indicates sensitivity encoding; MPFLAIR, magnetization-prepared FLAIR; FA, flip angle; TFE, turbo field echo.

been made in the same study population, up to $90 \%$ of patients with MS undergoing contrast-enhanced brain MR imaging at 7T demonstrated at least 1 enhancing focus. ${ }^{9}$ This result closely approximates the $89 \%$ of patients with MS reported to show some element of leptomeningeal inflammation at postmortem examination. ${ }^{10,11}$ Given this radiologic-pathologic concordance, it is conceivable that 7T 3D-FLAIR may soon provide a noninvasive in vivo method of detecting and accurately quantifying the extent of meningeal inflammation in patients with MS.

Meningeal enhancement was noted to be a persistent phenomenon in prior $3 \mathrm{~T}$ studies ${ }^{7}$; however, at 7T, where sensitivity for meningeal enhancement in MS appears to be significantly higher, it remains unknown whether smaller, more subtle foci of enhancement wax and wane in a predictable pattern across time or whether they remain longitudinally stable. Also unknown is the degree to which enhancement persistence with time is associated with previously described enhancement shape and morphology, including subarachnoid spread/fill and subarachnoid nodular patterns. ${ }^{7,9}$ Because prior studies have shown that the prevalence of meningeal enhancement varies with enhancement morphology, ${ }^{7,9}$ in this study, we hypothesized that persistence of meningeal enhancement in MS would vary on the basis of the morphology of enhancement as well as demographic and clinical factors such as treatment status, disease phenotype, and disability scores. Greater understanding of the imaging and clinical characteristics of meningeal enhancement is necessary if these features are to aid in the diagnosis of and prognosis for patients with MS.

\section{MATERIALS AND METHODS}

\section{Standard Protocol Approval and Informed Consent}

The institutional review boards at the authors' institutions approved this Health Insurance Portability and Accountability Actcompliant, prospective study. Written, informed consent was obtained from all participants.

\section{Participants}

Thirty-one volunteers, 26-61 years of age, with diagnoses of relapsing-remitting MS, secondary-progressive MS, or primaryprogressive MS according to the 2010 revised McDonald Criteria were recruited. ${ }^{12}$ Exclusion criteria included contraindications to contrast-enhanced MR imaging.

\section{MR Imaging Protocol}

Study participants were prospectively scanned at 2 time points approximately 1 year apart on a 7T Achieva scanner (Philips Healthcare, Best, the Netherlands) with a volume-transmit/32-channel head coil (Nova Medical, Wilmington, Massachusetts). Fifteen patients in the cohort were scanned at an additional third visit approximately 1 year later. Scans were obtained between September 9, 2014, and August 21, 2017. Dielectric padding was used for improved image homogeneity. ${ }^{13}$ Scanning parameters are listed in Table 1. Images were acquired before the administration of contrast and again after the intravenous administration of $0.1 \mathrm{mmol} / \mathrm{kg}$ of gadoteridol (ProHance; Bracco Diagnostics, Princeton, New Jersey). Magnetizationprepared rapid acquistion of 2 gradient echoes (MP2RAGE) images were initiated approximately 3 minutes after contrast administration, and magnetization-prepared FLAIR images were initiated approximately 20 minutes after contrast administration.

\section{Image Processing and Analysis}

MP2RAGE images were processed to create a T1-weighted image and a T1 map. ${ }^{14}$ Images were then manipulated using Medical Image Processing, Analysis, and Visualization (Version 7.2; http:// mipav.cit.nih.gov). Magnetization-prepared FLAIR images underwent N4 inhomogeneity correction before analysis. ${ }^{15}$ Pre- and postcontrast magnetization-prepared FLAIR images were registered to the precontrast T1 map. A magnetization-prepared FLAIR subtraction image was created by direct subtraction of the registered pre- and postcontrast images.

The magnetization-prepared FLAIR subtraction image, alongside the pre- and postcontrast magnetization-prepared FLAIR images, was reviewed by 2 independent raters (a postgraduate year 4 radiology resident and an academic MS neurologist) who were blinded to subject identity, disease state, and treatment regimen. Hyperintensities noted on the subtraction image were located on anatomic images and demarcated, if present, in the meningeal space on postcontrast images only. All foci were localized in 3 orthogonal planes before notation. When needed, coregistered MP2RAGE T1-weighted images were used for confirmation of anatomic locations. The pattern of enhancement was categorized on the basis of location and morphology and stratified into 1 of 4 subtypes. Subarachnoid spread/fill foci were characterized by the presence of contrast in the subarachnoid space distributed in an amorphous manner (Fig 1A). Subarachnoid nodular foci were characterized by small, round areas of contrast, usually $1-2$ voxels $(0.7-1.4 \mathrm{~mm})$ and were adherent to the pial surface (Fig $1 B$ ). Vessel wall enhancement was characterized by contrast outlining the outer margin of a large meningeal vessel with a signal void in the lumen of the vessel, often resulting in a characteristic tram-track appearance (Fig 1C). Dural foci were characterized by discrete regions of enhancement clearly situated along the dural surface without extension into the subarachnoid space (Fig $1 D)$. Following both independent reviews, a consensus review was performed under the supervision of an expert third rater (an academic neuroradiologist with 12 years of experience). After consensus review, follow-up images underwent linear registration (with $9 d f$ ) to baseline images. Consensus regions of contrast enhancement on baseline images were reviewed for their presence or absence on follow-up scans. The total number of foci per subject that persisted between scans was compared among different morphologies of meningeal enhancement and correlated 


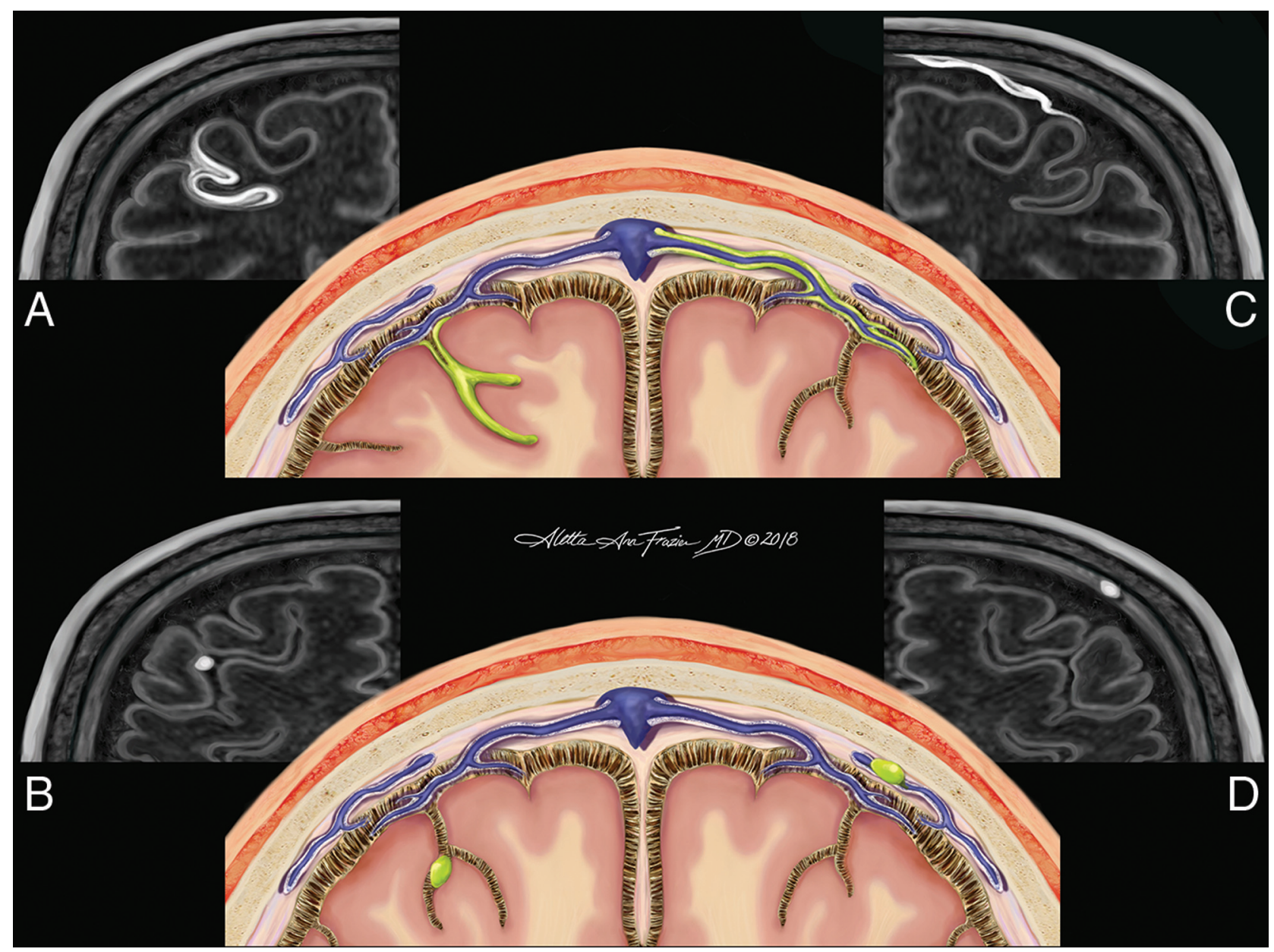

FIG 1. Original illustration depicting the 4 morphologies of meningeal enhancement seen in this analysis. Subarachnoid spread/fill pattern (represented by green in $A$ ) is an amorphous and ill-defined collection of contrast pooling within the cerebral sulci. The subarachnoid nodular pattern $(B)$ is defined as a punctate, discrete site of meningeal enhancement located within the cerebral sulci abutting the pial surface. The vessel wall pattern $(C)$ is characterized by extension of contrast along the outer margin of large meningeal vessels with a preserved internal flow void creating a characteristic tram-track appearance. The dural pattern $(D)$ is a circumscribed, rounded focus of contrast situated along the dural margin without extension into the subarachnoid space. The perivascular, tubular white structures (seen in schematics $A, B$, and $D$ ) represent the recently discovered meningeal lymphatic system. Reaccumulation of leaked contrast from the CSF into these meningeal lymph channels is a potential mechanism for the venous rim pattern (C).

with demographic and clinical data. Additionally, the proportion of baseline foci per subject that persisted to follow-up scans was also compared with morphologic, demographic, and clinical factors.

\section{Disability Measures}

The Kurtzke Expanded Disability Status Scale (EDSS) was used to characterize disability. ${ }^{16}$ EDSS progression was defined as an increase of the EDSS score at follow-up of $\geq 1.0$ if the baseline EDSS score was $<5.0$ or an increase of $\geq 0.5$ if the baseline EDSS score was $\geq 5.0$. The Modified Fatigue Impact Scale was used to assess MS-related fatigue. ${ }^{17,18}$ The Symbol Digit Modalities Test was used to assess cognitive functioning. ${ }^{19}$ These tests were administered at each study visit.

\section{Statistical Analysis}

Statistical analysis was performed in Stata 10.1 IC (StataCorp, College Station, Texas). Nonparametric testing was used due to the nonnormal distribution of data. We performed group comparisons for demographic and clinical variables using the Wilcoxon rank sum statistic. We computed the Spearman rank correlation for correlation testing. All statistical tests were performed with a significance threshold of $P<.05$. Due to the small sample size and exploratory nature of this study, adjustment for multiple comparisons was not performed.

\section{RESULTS}

We recruited 31 patients with MS; most had the relapsing-remitting MS phenotype $(n=21,68 \%)$, though 7 subjects had secondary-progressive MS (23\%) and 3 subjects had primary-progressive MS (10\%) (Table 2). No subject had a comorbid neuroinflammatory disorder. Most subjects were on diseasemodifying therapy $(n=25,81 \%)$. This was a relatively stable and moderately disabled patient population with a median of 0 (range, 0-3) relapses in the year before enrollment and a median Expanded Disability Status Scale score of 3 (range, 1-6.5). Enhancing white matter lesions were seen in 3 subjects on review of 
Table 2: Cohort baseline characteristics ${ }^{\mathrm{a}}$

\begin{tabular}{lc}
\hline \multicolumn{1}{c}{ Characteristics } \\
\hline Age at enrollment (yr) & $49(26-61)$ \\
Sex & $11 / 31$ Men $(35 \%), 20 / 31$ women (65\%) \\
Disease subtype at enrollment & $21 / 31(68 \%)$ RR, 7/31 (23\%) SP, \\
& $3 / 31(10 \%)$ PP \\
Disease duration at enrollment (mo) & $109(8-461)$ \\
Patients with new relapses in past 30 days & $1 / 31(3 \%)$ \\
No. of relapses in past year per subject & $0(0-3)$ \\
Modified Fatigue Impact Scale score at enrollment & $43(0-78)$ \\
Symbol Digit Modality Test at enrollment & $50(35-81)$ \\
Expanded Disability Status Scale score at enrollment & $3(1-6.5)$ \\
Immunomodulatory treatment status at baseline & \\
On treatment & $25 / 31(81 \%)$ \\
$\quad$ Not on treatment & $6 / 31(19 \%)$ \\
Treatment type at baseline & \\
Interferon & $3 / 25(12 \%)$ \\
Glatiramer & $6 / 25(24 \%)$ \\
Natalizumab & $2 / 25(8 \%)$ \\
Teriflunomide & $1 / 25(4 \%)$ \\
Fingolimod & $4 / 25(16 \%)$ \\
Dimethyl fumarate & $9 / 25(36 \%)$ \\
No. of subjects who switched between & $10 / 31$ \\
$\quad$ disease-modifying therapies from baseline to & \\
follow-up scans & \\
\hline
\end{tabular}

Note:-RR indicates relapsing-remitting MS; SP, secondary-progressive MS; PP, primary-progressive MS.

${ }^{a}$ Median values are shown with the range of observed values in parentheses.

Table 3: Anatomic distribution within the brain of enhancing meningeal foci at baseline

\begin{tabular}{lcc}
\hline \multicolumn{1}{c}{ Brain Region } & $\begin{array}{c}\text { No. of Foci } \\
\text { at Baseline }\end{array}$ & $\begin{array}{c}\text { Percentage of } \\
\text { Foci at Baseline }\end{array}$ \\
\hline Right frontal & 60 & 21.1 \\
Left frontal & 64 & 22.5 \\
Right parietal & 44 & 15.5 \\
Left parietal & 44 & 15.5 \\
Right occipital & 20 & 7.0 \\
Left occipital & 24 & 8.4 \\
Right temporal & 16 & 5.6 \\
Left temporal & 8 & 2.8 \\
Right cerebellum & 2 & 0.7 \\
Left cerebellum & 2 & 0.7 \\
\hline
\end{tabular}

T1-weighted images, with 2 subjects having 1 enhancing lesion and 1 subject having 2 enhancing lesions.

At baseline, a total of 284 enhancing foci were identified across all 31 subjects. Table 3 lists the anatomic distribution of these foci within the brain. Most (>98\%) of the observed foci were located supratentorially. Figure 2 shows the percentage of enhancing meningeal foci identified at baseline that persisted at later time points. Figures 3 and 4 provide examples of persisting and resolving enhancing meningeal foci from each group. Table 4 and Online Tables 1-3 compare the persistence of meningeal enhancement with demographic and clinical variables. We found no significant difference in the total number or proportion of longitudinally persistent enhancing meningeal foci between those on or off treatment or between those with progressive phenotypes (primary-progressive MS and secondary-progressive MS) versus a relapsing phenotype (relapsing-remitting MS). However, we did find significantly more $(P=.04)$ persistent foci in Expanded Disability Status Scale progressors (median, 12; range, 1-15) compared with those who were not progressors (median, 7.5; range, 1-24). We also observed a nonsignificant trend toward a negative

\section{DISCUSSION}

association ( $\rho=-0.31, P=.09$ ) between the proportion of persisting foci overall and the interval change in Symbol Digit Modalities Test scores at 1 year (On-line Table 3). Surprisingly and counterintuitively, we observed a positive correlation $(\rho=0.45, P=.01)$ between the proportion of enhancing meningeal foci that persisted at 1 year and baseline Symbol Digit Modalities Test scores (On-line Table 3). This association was driven by the correlation ( $\rho=0.48, P=.01$ ) between the proportion of subarachnoid spread/fill subtype that persisted at 1 year and baseline Symbol Digit Modalities Test scores. We also observed 15 foci of meningeal enhancement that developed in the interval between baseline and follow-up scans. The morphologies of these 15 foci were as follows: 6 subarachnoid spread/ fill, 4 subarachnoid nodular, 2 vessel wall, and 3 dural foci.

In this study, we catalogued 2 enhancement patterns described in prior analyses (subarachnoid spread/fill and subarachnoid nodular) ${ }^{7,9}$ in addition to describing 2 new patterns of meningeal enhancement for the first time: vessel wall enhancement and dural foci. Previous studies without precontrast comparison sequences excluded from consideration regions of postcontrast hyperintensity in/near the dural sinuses, large subarachnoid veins, and the basal meninges to reduce false-positives because these structures often manifest precontrast T1 or FLAIR hyperintensity. ${ }^{7,9}$ Using techniques similar to those in the recent investigation by Zivadinov et $\mathrm{al},{ }^{8}$ we coregistered and subtracted pre- and postcontrast magnetization-prepared FLAIR sequences in all cases. Given this protocol, we did not have to exclude any structures a priori, and we were confident in our ability to differentiate true vessel wall and dural foci enhancement from intrinsically increased signal. Which anatomic/pathologic substrates are represented by vessel wall and dural foci is unknown, but most interesting, both closely match what was recently described for visualization of meningeal lymphatics by FLAIR MR imaging. ${ }^{20-22}$ Thus, these findings may represent gadolinium absorption by lymphatic structures after leakage into the CSF. The accumulation of gadolinium signal alongside the outer wall of vessels in the vessel wall pattern is also very reminiscent of the expected location and direction of drainage of solutes from brain parenchyma along the recently described glymphatic system. ${ }^{23}$ Alternatively, it is also possible that vessel wall and dural foci could represent the reaccumulation, under hydrostatic pressure, of CSF-leaked gadolinium back into the venous system. They could also feasibly represent the actual sites of blood-brain and blood-CSF barrier disturbance secondary to ongoing inflammation. ${ }^{24}$ Because age- and sex-matched healthy controls were not used in this study, the specificity of dural and vessel wall enhancements to MS is unknown. Future 
work is needed to determine whether such findings are specific to MS, neuroinflammatory disease in general, or are seen in all patients.

We found no significant difference in the total number or proportion of persisting meningeal enhancement per subject between those on treatment and those off treatment. Lack of a significant difference between subjects on/off treatment may, in part, be explained by the low statistical power of our study because a relatively small number of untreated subjects were included.
However, the lack of difference is not surprising because prior studies have also failed to show differences in meningeal enhancement between those who are and are not taking disease-modifying medications. ${ }^{7}$ Our data reinforce the notion that current immunomodulatory medications may not adequately control meningeal inflammation. Of note, none of our subjects received a course of corticosteroids during the study. However, 10 of 31 subjects in this cohort switched between disease-modifying therapies from baseline to follow-up scans, including 2 subjects who changed to

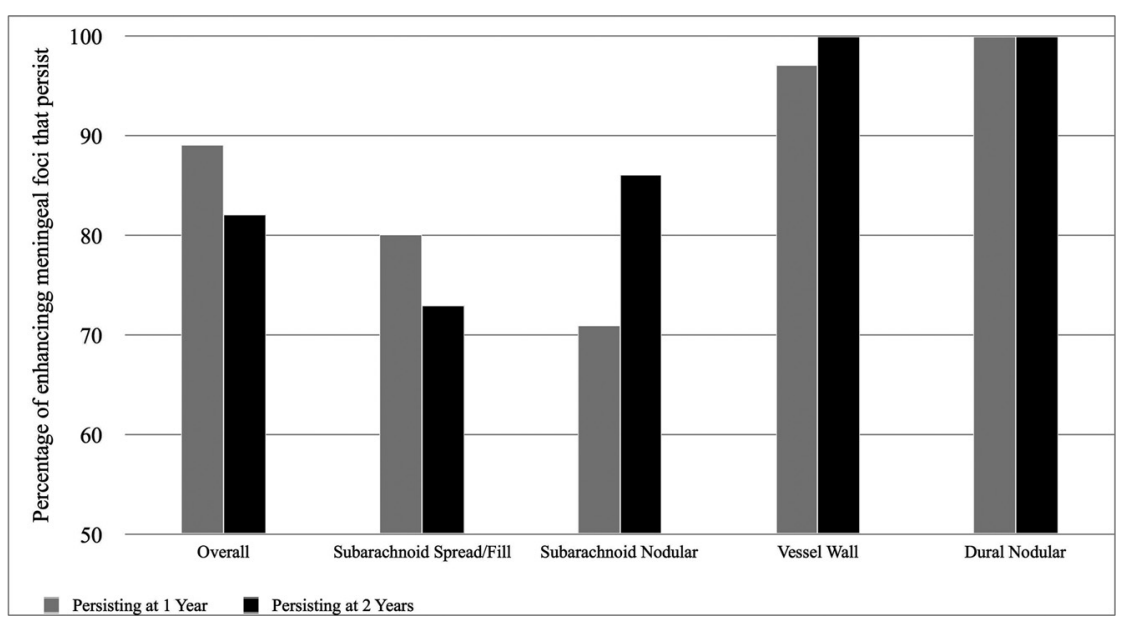

FIG 2. Graph displaying the percentages of baseline enhancing meningeal foci that persist 1 year later (gray bars) and 2 years later (black bars). All 31 participants were scanned at baseline and at 1 year, but 2-year data are limited to 15 participants. At 1 year, persistence was noted in 253/284 (89\%) overall foci, 91/114 (80\%) subarachnoid spread/fill foci, 10/14 (71\%) subarachnoid nodular foci, 104/107 (97\%) vessel wall foci, and 46/46 (100\%) dural nodular foci. At 2 years, persistence was noted in 132/161 (82\%) overall foci, 45/62 (73\%) subarachnoid spread/fill foci, 6/7 (86\%) subarachnoid nodular foci, $55 / 55$ (100\%) vessel wall foci, and $34 / 34$ (100\%) dural foci. rituximab and 1 subject who switched to alemtuzumab-both monoclonal antibodies that impact B-cell function. Despite such changes, most foci remained stable. Given the sample size of this report, we would not want to comment on the persistence (or lack thereof) of foci with individual therapy changes because conclusions from 1 or 2 examples would not be generalizable. Future comparative studies are needed to determine whether changes in any specific disease-modifying therapies or monoclonal regimens alter the longitudinal persistence of meningeal enhancement.

Surprisingly, we did not detect a significant difference in the persistence of meningeal enhancement between MS subjects with progressive phenotypes (primary-progressive MS and secondary-progressive MS) and those with re-

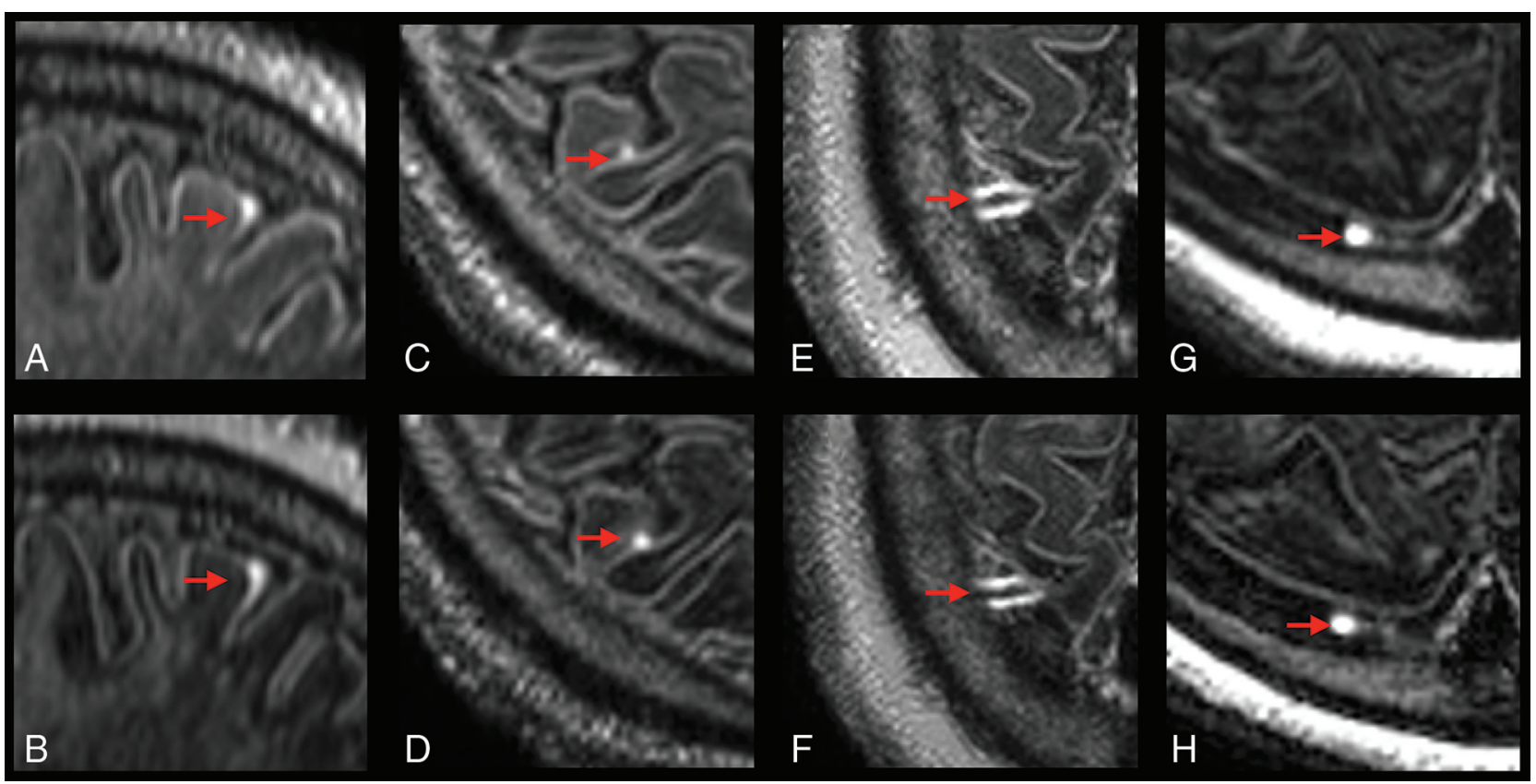

FIG 3. Examples of persisting foci of meningeal enhancement on delayed postcontrast FLAIR at 7T. Sagittal reformatted images show subarachnoid spread/fill enhancement that persists from December 15, 2015 (A), to March 3, 2017 (B), in a 58-year-old woman with relapsing-remitting MS. Axial images show subarachnoid nodular enhancement that persists from October 8, 2014 (C), to March 3, 2017 (D), in a 49-year-old man with relapsing-remitting MS. Axial images show vessel wall enhancement that persists from March 14, 2016 (E), to April 4, 2017 (F), in a 57-year-old man with primary-progressive MS. Axial images show dural enhancement that persists from May 9, 2016 (G), to May 31, 2017 (H), in a 44-year-old woman with secondary-progressive MS. Note that no intrinsic signal was observed in these locations on precontrast acquisitions (not shown). 
lapsing-remitting MS. This finding runs counter to the previously proposed theory that meningeal enhancement may be a substrate specific to progressive MS, with the associated cortical demyelination and volume loss representing a distinctly late marker of disease. ${ }^{1,7}$ Indeed, previous studies have shown that the presence of leptomeningeal enhancement at $3 \mathrm{~T}$ was 1.7 -fold higher in progressive MS compared with relapsing-remitting MS, and postmortem findings of meningeal inflammation were more profound in those with secondary-progressive MS. ${ }^{1,7}$ However, our 7T data showed no difference in the frequency of longitudinal persistence of meningeal enhancement between patients with MS with progressive and relapsing phenotypes.

Although the persistence of enhancing foci was not related to clinical phenotype, we did detect a significant relationship between the persistence of foci (especially subarachnoid spread/fill

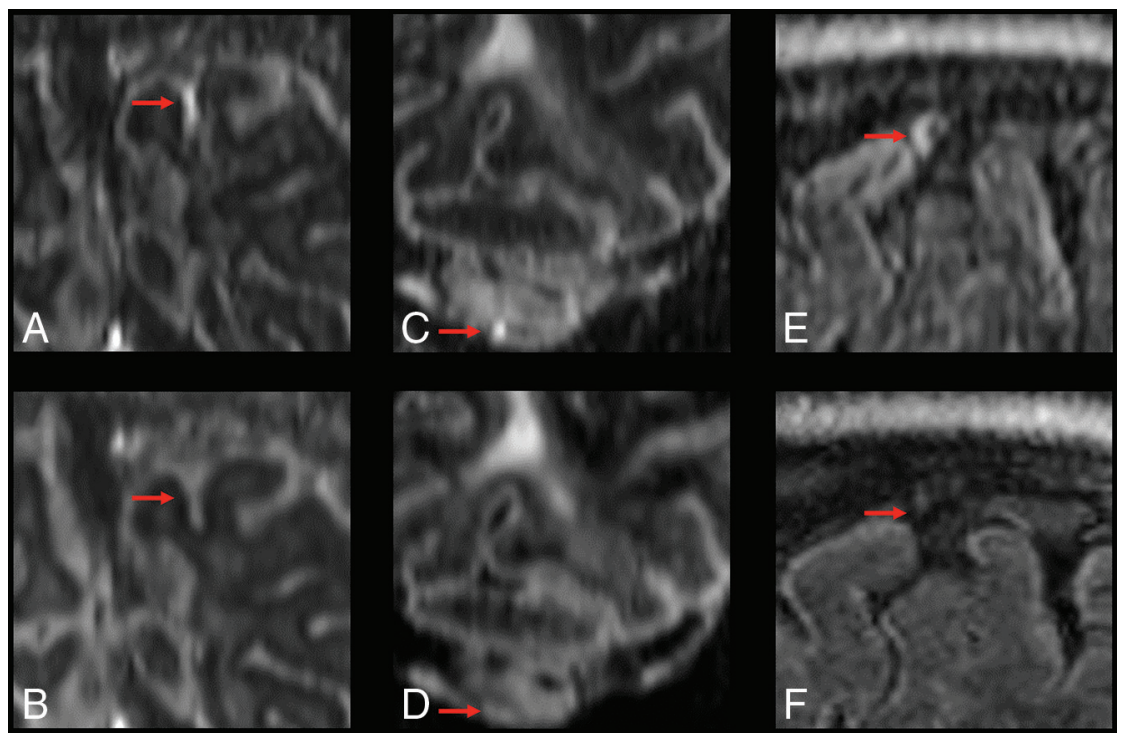

FIG 4. Examples of resolving foci of meningeal enhancement on delayed postcontrast FLAIR at 7T. Coronal reformatted images show subarachnoid spread/fill enhancement that resolves between October 23, $2014(A)$, and February 26, $2016(B)$, in a 49-year-old woman with relapsingremitting MS. Coronal reformatted images show subarachnoid nodular enhancement within the cerebellar folia that resolves between October 8, 2014 (C), and February 19, $2016(D)$, in a 49-yearold man with relapsing-remitting MS. Sagittal formatted images show vessel wall enhancement that resolves from May 9, $2016(E)$, to May 31, 2017 (F), in a 44-year-old woman with secondaryprogressive MS. Note that no foci of meningeal enhancement classified as a dural subtype resolved in this study.

Table 4: Wilcoxon rank sum test for longitudinal persistence of meningeal enhancement versus demographic and clinical factors ${ }^{a}$

\begin{tabular}{|c|c|c|c|c|c|c|}
\hline & $\begin{array}{c}\text { On } \\
\text { Treatment } \\
(n=25)\end{array}$ & $\begin{array}{l}\text { Not on } \\
\text { Treatment } \\
(n=6)\end{array}$ & $\begin{array}{l}\text { Progressive } \\
\text { MS }(n=10)\end{array}$ & $\begin{array}{c}\text { Nonprogressive } \\
\text { MS }(n=21)\end{array}$ & $\begin{array}{c}\text { EDSS } \\
\text { Progressor at } \\
1 \mathrm{yr}^{\mathrm{b}}(n=7)\end{array}$ & $\begin{array}{c}\text { EDSS } \\
\text { Nonprogressor } \\
\text { at } 1 \mathrm{yr}^{\mathrm{b}}(n=24)\end{array}$ \\
\hline $\begin{array}{l}\text { Total No. of overall foci persisting at } 1 \mathrm{yr} \\
\text { per subject }\end{array}$ & $9(1-24)$ & $9(1-15)$ & $8(1-24)$ & $9(1-15)$ & $12(1-15)$ & $7.5(1-24)^{c}$ \\
\hline $\begin{array}{l}\text { Total No. of subarachnoid spread/fill foci } \\
\text { persisting at } 1 \text { yr per subject }\end{array}$ & $2(0-9)$ & $2(0-6)$ & $2(0-9)$ & $2(0-9)$ & $5(1-9)$ & $2(0-9)$ \\
\hline $\begin{array}{l}\text { Total No. of subarachnoid nodular foci } \\
\text { persisting at } 1 \text { yr per subject }\end{array}$ & $0(0-2)$ & $0(0-1)$ & $0(0-0)$ & $0(0-2)^{c}$ & $0(0-0)$ & $0(0-2)$ \\
\hline $\begin{array}{l}\text { Total No. of vessel wall foci persisting at } \\
1 \mathrm{yr} \text { per subject }\end{array}$ & $3(0-11)$ & $3.5(1-6)$ & $2(0-6)$ & $3(0-11)$ & $3(0-11)$ & $3(0-7)$ \\
\hline $\begin{array}{l}\text { Total No. of dural foci persisting at } 1 \mathrm{yr} \\
\text { per subject }\end{array}$ & $1(0-9)$ & $1(0-6)$ & $1(0-9)$ & $1(0-6)$ & $1(0-6)$ & $1(0-9)$ \\
\hline
\end{tabular}

${ }^{a}$ Median values are listed with the range of observed values in parentheses.

${ }^{b}$ Criteria for EDSS progressor status is listed in the "Materials and Methods" section.

${ }^{c} p<.05$. 
important new insight into the longitudinal activity of meningeal enhancement in MS.

\section{CONCLUSIONS}

Here we describe the results of a prospective, systematic investigation into the longitudinal persistence of meningeal enhancement in MS using 7T 3D-FLAIR. Given our pre- and postcontrast techniques, we are able to include, for the first time, vessel wall and dural foci subtypes, which persist most frequently; their appearance very closely matches recent descriptions of meningeal lymphatics or the glial lymphatics system. ${ }^{19-22}$ Longitudinal persistence of meningeal enhancement is not significantly different between those on or off immunomodulatory treatment, and there is not a significant difference in the rates of longitudinal persistence between those with progressive clinical phenotypes (primary-progressive MS and secondary-progressive MS) and those without a progressive clinical phenotype (relapsing-remitting MS). However, there is a significantly increased number of persistent foci in subjects who have worsening Expanded Disability Status Scale scores at 1 year compared with those who do not, suggesting that persistently enhancing meningeal foci may be an in vivo imaging marker for ongoing meningeal inflammation causing clinical progression.

Disclosures: Samuel N. Jonas-UNRELATED: Support for Travel to Meetings for the Study or Other Purposes: 2018 Radiological Society of North America Resident Travel Grant, Comments: \$500; Provision of Writing Assistance, Medicines, Equipment, or Administrative Support: Department of Radiology Pagination Editor. Izlem Izbudak-UNRELATED: Consultancy: Alexion, Comments: Neuromyelitis Optica Relapse Adjudication Committee MRI reads; Grants/Grants Pending: Siemens, Biogen, Comments: Siemens DTI of the spinal cord prospective research study, Biogen MS PATHS MRI scan-rescan study. Aletta A. Frazier—UNRELATED: Consultancy: American Institute for Radiologic Pathology, Comments: Section Chief, Cardiovascular Radiology; Associate Chief, Chest Radiology, American Institute for Radiologic Pathology, a program of the American College of Radiology, Silver Spring, Maryland; Employment: Clinical Professor of Radiology, University of Maryland School of Medicine, Comments: Clinical Professor of Diagnostic Radiology and Nuclear Medicine, University of Maryland School of Medicine, Baltimore, Maryland. Daniel M. Harrison—RELATED: Grant: EMD-Serono, Comments: research grant*; UNRELATED: Consultancy: EMD-Serono, Genentech, Genzyme, Biogen, Comments: honoraria for 1-time advisory board meetings; Payment for Lectures Including Service on Speakers Bureaus: Houston Methodist Hospital; Other: American College of Physicians, Comments: textbook chapter writing and multiple-choice question development for Medical Knowledge Self Assessment Program (MKSAP). *Money paid to the institution.

\section{REFERENCES}

1. Magliozzi R, Howell O, Vora A, et al. Meningeal B-cell follicles in secondary progressive multiple sclerosis associate with early onset of disease and severe cortical pathology. Brain J Neurol 2007;130 (Pt 4):1089-104 Medline

2. Lucchinetti CF, Popescu BF, Bunyan RF, et al. Inflammatory cortical demyelination in early multiple sclerosis. N Engl J Med 2011;365: 2188-97 CrossRef Medline

3. Choi SR, Howell OW, Carassiti D, et al. Meningeal inflammation plays a role in the pathology of primary progressive multiple sclerosis. Brain J Neurol 2012;135(Pt 10):2925-37 CrossRef Medline

4. Serafini B, Rosicarelli B, Magliozzi R, et al. Detection of ectopic B-cell follicles with germinal centers in the meninges of patients with secondary progressive multiple sclerosis. Brain Pathol 2004;14:164-74 CrossRef Medline

5. Kowarik MC, Cepok S, Sellner J, et al. CXCL13 is the major determi- nant for B cell recruitment to the CSF during neuroinflammation. J Neuroinflammation 2012;9:93 CrossRef Medline

6. Nielsen AS, Kinkel RP, Tinelli E, et al. Focal cortical lesion detection in multiple sclerosis: 3 Tesla DIR versus 7 Tesla FLASH-T2. J Magn Reson Imaging 2012;35:537-42 CrossRef Medline

7. Absinta M, Vuolo L, Rao A, et al. Gadolinium-based MRI characterization of leptomeningeal inflammation in multiple sclerosis. $\mathrm{Neu}$ rology 2015;85:18-28 CrossRef Medline

8. Zivadinov R, Ramasamy DP, Hagemeier J, et al. Evaluation of leptomeningeal contrast enhancement using pre-and postcontrast subtraction 3D-FLAIR imaging in multiple sclerosis. AJNR Am J Neuroradiol 2018;39:642-47 CrossRef Medline

9. Harrison DM, Wang KY, Fiol J, et al. Leptomeningeal enhancement at $7 \mathrm{~T}$ in multiple sclerosis: frequency, morphology, and relationship to cortical volume. J Neuroimaging 2017;27:461-68 CrossRef Medline

10. Howell OW, Reeves CA, Nicholas R, et al. Meningeal inflammation is widespread and linked to cortical pathology in multiple sclerosis. Brain J Neurol 2011;134(Pt 9):2755-71 CrossRef Medline

11. Howell OW, Schulz-Trieglaff EK, Carassiti D, et al. Extensive grey matter pathology in the cerebellum in multiple sclerosis is linked to inflammation in the subarachnoid space. Neuropathol Appl Neurobiol 2015;41:798-813 CrossRef Medline

12. Polman CH, Reingold SC, Banwell B, et al. Diagnostic criteria for multiple sclerosis: 2010 revisions to the McDonald criteria. Ann Neurol 2011;69:292-302 CrossRef Medline

13. Haines K, Smith NB, Webb AG. New high dielectric constant materials for tailoring the $\mathrm{B} 1+$ distribution at high magnetic fields. $J$ Magn Reson 2010;203:323-27 CrossRef Medline

14. Marques JP, Kober T, Krueger G, et al. MP2RAGE, a self bias-field corrected sequence for improved segmentation and T1-mapping at high field. Neuroimage 2010;49:1271-81 CrossRef Medline

15. Tustison NJ, Avants BB, Cook PA, et al. N4ITK: improved N3 bias correction. IEEE Trans Med Imaging 2010;29:1310-20 CrossRef Medline

16. Kurtzke JF. Rating neurologic impairment in multiple sclerosis: an expanded disability status scale (EDSS). Neurology 1983;33:1444-52 CrossRef Medline

17. Multiple Sclerosis Council for Clinical Practice Guidelines. Fatigue and multiple sclerosis: evidence-based management strategies for fatigue in multiple sclerosis. Paralyzed Veterans of America 1998:1-33

18. Flachenecker P, Kumpfel T, Kallmann B, et al. Fatigue in multiple sclerosis: a comparison of different rating scales and correlation to clinical parameters. Mult Scler 2002;8:523-26 CrossRef Medline

19. Smith A. Symbol Digit Modalities Test: Manual. Los Angeles: Western Psychological Services; 1973

20. Aspelund A, Antila S, Proulx ST, et al. A dural lymphatic vascular system that drains brain interstitial fluid and macromolecules. $J$ Exp Med 2015;212:991-99 CrossRef Medline

21. Raper D, Louveau A, Kipnis J. How do meningeal lymphatic vessels drain the CNS? Trends Neurosci 2016;39:581-86 CrossRef Medline

22. Absinta M, Ha SK, Nair G, et al. Human and nonhuman primate meninges harbor lymphatic vessels that can be visualized noninvasively by MRI. Elif 2017;6 CrossRef Medline

23. Plog BA, Nedergaard $M$. The glymphatic system in central nervous system health and disease: past, present, and future. Annu Rev Pathol 2018;13:379-94 CrossRef Medline

24. Ortiz GG, Pacheco-Moisés FP, Macías-Islas MA, et al. Role of the blood-brain barrier in multiple sclerosis. Arch Med Res 2014;45: 687-97 CrossRef Medline

25. Zivadinov R, Ramasamy DP, Vaneckova M, et al. Leptomeningeal contrast enhancement is associated with progression of cortical atrophy in MS: a retrospective, pilot, observational longitudinal study. Mult Scler 2017;23:1336-45 CrossRef Medline

26. Absinta M, Cortese IC, Vuolo L, et al. Leptomeningeal gadolinium enhancement across the spectrum of chronic neuroinflammatory diseases. Neurology 2017;88:1439-44 CrossRef Medline 